\title{
Antibiotic Profile of Bacterial Species Isolated from Broiler Chickens with Cellulitis
}

\author{
Mohamed M. Amer ${ }^{1 *}$, Hoda M. Mekky ${ }^{2}$, Hanaa S. Fedawy ${ }^{2}$, Kh. M. Elbayoumi ${ }^{2}$ and Dalia M. Sedeek ${ }^{2}$ \\ ${ }^{1}$ Department of Poultry Diseases, Faculty Veterinary Medicine, Cairo University, P.O. 12211, Giza, Egypt \\ ${ }^{2}$ Poultry Diseases Department, Veterinary Research Division, National Research Centre, P.O. 12622, Giza, Egypt \\ *Corresponding author's Email: profdramer@yahoo.com; (1DORCiD: 0000-0001-8965-7698
}

\begin{abstract}
The present study was carried out to isolate and identify the bacterial agents involved in field cases of avian cellulitis in broiler chickens and also to examine isolated bacteria for antibiotic susceptibility. The study was applied on 290 broiler chickens, aged 30-35 days, suffered from cellulitis (65 with head and 225 body lesions) to isolate bacterial agents. All obtained isolates were identified and tested for the pathogenicity based on Congo red assay. Disc diffusion test was used to study the sensitivity pattern of bacterial isolates with determination of multiple antibiotic resistance index. Results revealed that all head and $91.5 \%$ of body samples were positive on bacteriological examination. E. coli was the most prevalent isolate $(45.2 \%)$, followed by staphylococci $(33.2 \%)$, Clostridia $(5.4 \%)$ streptococci (5.1\%), Proteus mirabilis (4.4\%), Enterobacter spp. (3.2\%), Pseudomonas aeruginosa (2.2\%), and Aeromonas spp. (1.2\%). Congo red binding test was positive for P. aeruginosa (100\%), Clostridia (72.7\%), E. coli (65.8\%), staphylococci (62.2\%), Aeromonas spp. (60\%), P. mirabilis (38.9\%), Enterobacter spp. (38.5\%) and streptococci $(33.3 \%)$. Serological typing of E. coli identified nine O serotypes, with high predominance of O78 $(19 \%)$. On antibiotic susceptibility profiling, E. coli isolates demonstrated $83.1-92.9 \%$ resistance to chloramphenicol, tetracycline, and enrofloxacin. Staphylococci isolates showed high resistance to ampicillin (97.0\%) and clindamycin (82.9\%). Clostridial and Aeromonas spp. isolates showed $100 \%$ resistant to tetracycline, enrofloxacin, and cefotaxime. Enterobacter spp. showed $100 \%$ resistance to chloramphenicol and cefotaxime. $P$. aeruginosa had $100 \%$ resistance to tetracycline and enrofloxacin. Also, streptococci isolates showed $100 \%$ resistance to erythromycin. Totally, $56.3 \%$ bacterial isolates were multidrug-resistant, $23.8 \%$ extensively drugresistant and $1.5 \%$ pan drug-resistant. The present study concluded that $E$. coli is the most predominant pathogen involved in cellulitis, particularly $\mathrm{O} 78$ serotype. In addition, this study demonstrated high prevalence of multidrugresistant bacteria among isolates, particularly against commonly used antibiotics. Therefore, it is recommended to use antibiotic sensitivity tests and accurate therapeutic doses to efficiently treat and control bacterial infections in poultry.
\end{abstract}

Key words: Antibacterial susceptibility, Bacterial isolates, Broiler, Cellulitis, Sensitivity classes.

\section{INTRODUCTION}

Avian cellulitis, known as necrotic dermatitis, is one of the most prevalent infections in broiler chickens and has been reported from many countries around the world with a developed poultry industry. Birds with cellulitis often do not show clinical symptoms (Norton 1997). Macroscopically, cellulitis lesions are characterized by deposition of yellowish fibrin under discolored or thickened skin. In cases of involvement of the skin surface, there may be an oozing exudate over the skin "waffle skin", and infections are most common over the thigh muscle, breast, legs, abdomen, head and neck (Randall et al., 1984; Fallavena et al., 2000; Gomis et al., 2000). Clinically, cellulitis can be seen in affected chicken if infection occurs in the head region, and it looks as swollen head syndrome in 5-6 weeks old chickens (Morley and Thomson 1984), while affection of other body sites can be only detected accidentally in post mortem examination or during inspection in slaughterhouse (Bianco et al., 2016). At processing, the presence of subcutaneous fibrinonecrotic plaques accompanied by inflammation of overlying skin lead to total or partial carcass rejection (de Brito et al., 2003). Hence, economic losses are mainly due to increased condemnation rate and downgrading of affected carcasses (Bianco et al., 2016). In the USA, losses due to cellulitis are estimated to be 40-50 million dollars annually and cause up to $30 \%$ of total carcass condemnation in broilers (Norton, 1997). In Canada, 0.8\% of slaughtered broilers were condemned for cellulitis in 2004 (Paniago, 2009). In Brazil, cellulitis lesions are estimated to cause at least 18 thousand tons meat losses in 2011 (Barbieri et al., 2013). Unfortunately, in Egypt, there is no accurate data about losses attributed to this problem.

Avian cellulitis is mainly caused by bacterial agents such as Actinomyces pyogenes, Pasteurella multocida, Pseudomonas aeruginosa, Streptococcus dysagalactae, Staphylococcus spp., Aeromonas spp., Proteus vulgaris, and Enterobacteriaceae through the invasion of subcutaneous tissue. Escherichia coli is the predominant organism (Barros et al., 2013) colonized in the subcutaneous tissues in avian cellulitis (Peighambari et al., 1995; Gomis et al., 2000; Derakhshanfar and Ghanbarpour 2002). Cellulitis lesions caused by loss of skin integrity (as a predisposing factor) in a 
susceptible host infected with bacterial agents. Trauma to the skin is essential for the development of avian cellulitis, as there are no records for lesions in chickens with non-traumatized skin (Peighambari et al.1995). Injurious behavior, cannibalism, biting insects, poor litter conditions, foot problems that lead to long sitting of birds, immunodeficiency and systemic infections are considered as predisposing factors in avian cellulitis (Rosenberger et al., 1975; Peighambari et al. 1995; Norton 1997; Wang et al., 2005; Bianco et al., 2016).

The development of antimicrobial resistance among bacteria makes antimicrobial susceptibility tests essential to detect drug resistance and to identify susceptibility to drugs for proper treatment of particular infections (Jorgensen and Ferraro, 2009). For interpretation of antimicrobial susceptibility, isolates have Multiple Antibiotic Resistance (MAR) index $\geq 0.3$ are either multidrug-resistant (MDR) (resistant to 3 or 4 class of antibiotics) or expanded drugresistant (resistant to more than five antibiotics classes). The most resistance patterns observed among the MDR isolates indicate that these isolates originate from a high-risk source of contamination where antibiotics are often used (Christopher et al., 2013).

The present study aimed to isolate and identify the bacterial agents involved in field cases of avian cellulitis in broiler chicken as well as examine the isolated bacteria for antibiotic susceptibility.

\section{MATERIAL AND METHODS}

\section{Ethical approval}

This study was approved by the Ethical Committee for Medical Research at the National Research Centre, Egypt and in accordance with local laws and regulations.

\section{Chicken flocks}

This study was conducted on 290 broiler chickens, ranged from 30 to 35 days old, suffered from cellulitis (65 with head and 225 body lesions). The chickens were collected from 45 flocks, with an average stock density of 21,000 chicken/house, located in full integrated poultry farms in Giza, Behera, and Sharkia governorates, Egypt from March 2017 to March 2019. Birds with head cellulitis were diagnosed at farms while body cellulitis was identified in carcasses at slaughterhouses.

Flocks with head cellulitis (13 flocks) were sampled directly at the farm (5 birds/flock) and resampled again at slaughterhouse after de-feathering ( 5 carcasses with body lesions/flock). The rest of the chicken flocks (32 flocks) were sampled at slaughterhouse after de-feathering (5 carcasses with body lesions/flock). All birds were subjected to postmortem examination and samples collected from cellulitis lesion for bacteriological examination.

\section{Bacteriological examination}

\section{Sampling}

For bacteriological examination, sterile swabs were individually collected from subcutaneous exudates of cellulitis lesions ( 3 swabs/bird). The collected samples were labeled and transported in cool boxes to the laboratory.

\section{Culture media}

Bacterial enrichment was done using nutrient broth, tryptone soya broth, and LB broth as liquid media at $37^{\circ} \mathrm{C}$ for 18 hours. Further bacterial isolation and identification were carried out using selective and differential media, including Salmonella-Shigella agar and MacConkey agar (for Enterobacteriaceae), nutrient agar and blood agar (for Gram-positive bacteria) and Pseudomonas isolation agar (for $P$. aeruginosa), which were prepared and used according to Collee et al., (1996); Forbes et al., (2002) and Greenwood et al., (2005).

\section{Bacterial isolation and identification}

After initial enrichment, a loopful of the enriched broth was streaked on solid media and incubated at the recommended temperature and time, then examined for bacterial growth according to Quinn et al., (1994) and Collee et al., (1996). The suspected colonies were picked up, purified and kept in semi-solid agar for further morphological and biochemical analysis (Konemann et al., 1992; Quinn et al., 2002). Identification and characterization of the obtained isolates were done according to colony morphology and Gram staining (Forbes et al., 2002; Greenwood et al., 2005). Proper biochemical characterization was done using API identification kits (API System, France) and was analyzed using Bergey's manual of systematic bacteriology (Sneath et al., 1986).

\section{Serological typing of $E$. coli}

The obtained E. coli isolates were subjected to serological identification according to Edward and Ewing (1972) using polyvalent and monovalent diagnostic E. coli antisera (Deben Diagnostics Ltd., UK) through the application of slide agglutination test. 


\section{In vitro pathogenicity test}

The purified isolates of all bacterial spp. were tested based on Congo red (CR) dye-binding assay in order to differentiate between pathogenic and non-pathogenic microorganisms according to Berkhoff and Vinal (1986). Each isolate was cultured on a separate plate of Trypticase soy agar supplemented with $0.003 \%$ CR dye (Sigma, UK) and $0.15 \%$ bile salts. Plates were incubated aerobically at $37^{\circ} \mathrm{C}$ for 24 hours. Then, the cultures were left at room temperature for an additional 48 hours to obtain clear results. The appearance of deep brick-red colonies between 24 and 72 hours of incubation was recorded as positive (CR+).

\section{Antibiogram}

\section{Antibiotic discs}

The following 10 antibiotic discs were used: gentamycin $10 \mu \mathrm{g} / \mathrm{ml}(\mathrm{CN})$, oxacillin $30 \mu \mathrm{g} / \mathrm{ml}$ (OX), erythromycin 15 $\mu \mathrm{g} / \mathrm{ml}$ (ERI), chloramphenicol $30 \mu \mathrm{g} / \mathrm{ml}$ (C30), tetracycline $30 \mu \mathrm{g} / \mathrm{ml}$ (T30), clindamycin $2 \mu \mathrm{g} / \mathrm{ml}$ (DA), enrofloxacin 5 $\mu \mathrm{g} / \mathrm{ml}$ (ENR), ampicillin $10 \mu \mathrm{g} / \mathrm{ml}$ (AMP), cefotaxime $30 \mu \mathrm{g} / \mathrm{ml}$ (CTX) and vancomycin $30 \mu \mathrm{g} / \mathrm{ml}$ (VA), representing antibacterial categories of aminoglycosides, $b$-lactams, macrolides, phenicols, tetracycline, lincosamides, fluoroquinolones, penicillin, cephalosporin and glycopeptides, respectively. The selection of disk concentrations and interpretations of zone diameters were done as recommended by the manufacturers (Difco Laboratories, Detroit, MI, USA) and CLSI (2016).

\section{Antibiotic susceptibility test}

Antibiotic susceptibility of the identified isolates was determined using the disc agar diffusion test according to Watts (2008) and CLSI (2016). Separate and similar colonies on solid media plate were emulsified in $3 \mathrm{ml}$ of normal saline and the turbidity was adjusted to $0.5 \mathrm{McFarland}$ standard. Using sterile swab sticks, the Muller Hinton agar plates, $9 \mathrm{~cm}$-diameter, were inoculated with the bacterial suspension by streaking the surface of the agar and rotating the plate to ensure even distribution. The inoculated plates were allowed to dry at room temperature for 10 minutes and then antibiotic discs were placed on the surface of the agar. The plates were left at room temperature for the pre-diffusion time before aerobic incubation at $37^{\circ} \mathrm{C}$ for $16-18$ hours. Growth inhibition zones were measured to the nearest millimeter and isolates classified as sensitive, intermediate and resistant based on CLSI (2016).

\section{Determination of multiple antibiotic resistances index}

The MAR index was determined by the following formula: MAR $=\mathrm{a} / \mathrm{b}$

where $\mathrm{a}$ is the number of antibiotics to which the test isolate was resistant; and $\mathrm{b}$ is the total number of antibiotics that the test isolate has been evaluated for susceptibility (Krumperman, 1983; Paul et al., 1997). According to standardized international terminology created by European Centre for Disease Control and Centre for Disease Control and Prevention, Atlanta, the MDR bacteria were defined as non-susceptible to at least one agent in three or more antimicrobial categories, Extensively Drug-Resistant (XDR) bacteria were defined as non-susceptible to at least one agent in all but two or fewer antimicrobial categories, and Pan Drug-Resistant (PDR) bacteria were defined as nonsusceptible to all agents in all antimicrobial categories (Magiorakos et al., 2012).

\section{RESULT AND DISCUSSION}

In the present study, the mortality rate in sampled flocks ranged from 5.6 to $10.5 \%$. The number of transported chickens to slaughterhouse ranged from 18795 to 19845 per house. The incidence rate of head cellulitis in 13 flocks was $0.2-1.26$ percent while the rate of rejected carcasses due to cellulitis after de-feathering was 0.9-1.7 percent. These findings are consistent with the data recorded by Amini et al. (2015) who reported the average overall condemnation rate for 16 broiler farms processed by two processing plants was $1.4 \%$; while the average total condemnation rate due to cellulitis was $0.83 \%$ over the 12 -months period. Also, cellulitis was recorded in 126 condemned carcasses and 272 broilers dead on their own and reported as the main cause of condemnation in 13 broiler flocks between 2014 and 2016 (Poulsen, 2018).

In the present study, 290 cellulitis samples were represented by 65 head cellulitis and 225 body cellulitis. Concerning body cellulitis samples, the most common lesions were located as diffuse lesions in general body region, followed by the abdomen and the thigh. The lowest incidence was in the back region (Table 1). These findings are similar to those described in previous studies (Messier et al., 1993; Fallavena et al., 2000; Alves et al., 2007). One study recorded that well-characterized lesions were generally located in the thigh, back and cloacal area (Alves et al., 2007).

Cellulitis lesions appeared as irregular and thick skin with dark to brown discoloration, either circumscribed localized or generalized throughout the body (Figure 1). Skinning of these lesions revealed the existence of the characteristic yellowish to green subcutaneous exudates which were fibrinous, serosanguineous, or suppurative (Figure 
2). The detected exudate was found to extend to the subcutaneous layers in some cases. Fibrinous to caseous pericarditis, airsacculitis, bursal and kidney lesions were recorded in some cases.

In the present study, all head cellulitis samples and $91.5 \%$ of body cellulitis samples had positive results for bacterial examination where 407 bacterial isolates were recovered (Table 1). The most prevalent isolated bacteria were E. coli (45.2\%), followed by Staphylococcus spp. (33.2\%), Clostridia (5.4\%), Streptococcus spp. (5.1\%), Proteus mirabilis (4.4\%), Enterobacter spp. (3.2\%), P. aeruginosa (2.2\%) and Aeromonas spp. (1.2\%) (Table 2). Similar results were obtained by Santos et al. (2014) who isolated 25 avian cellulitis lesion samples, of which 11 isolates were E. coli strains, 9 were S. epidermidis strains, 7 were Proteus mirabilis, and 3 were Manheimia haemolytica. In another study, bacteriological and mycological examination for 40 cellulitis lesions from 28 poultry farms indicated that the most prevalent bacteria were E. coli $(96.4 \%)$, followed by Citrobacter spp. (10.7\%), Proteus vulgaris (7.1\%), Staphylococcus spp., Streptococcus spp., Candida albicans, P. aeruginosa, Klebsiella spp., Serratia spp., Penicillium spp. and Aspergillus spp. (Brito et al., 2011). Also, in previous studies, a number of bacteria such as Aeromonas, Enterobacter, Pasteurella, Proteus, Pseudomonas, Streptococcus and E. coli, as the most predominant bacteria, were recovered from cellulitis lesions (Norton et al., 1997; Gomis et al., 2002; Shawki et al., 2017). However, in some studies, only E. coli was isolated from all (100\%) broiler carcasses-affected cellulitis (Andrade, 2005; Vieira et al., 2006).

Results from CR binding test indicated that E. coli (65.8\%), Staphylococcus (62.2\%), Clostridia (72.7\%), Aeromonas spp. (60\%), Enterobacter spp. (38.5\%), Proteus mirabilis (38.9\%), P. aeruginosa (100\%), and Streptococcus spp. (33.3\%) were positive (Table 2). CR binding activity test was applied in order to distinguish pathogenic from nonpathogenic strains of bacteria in vitro (Berkhoff and Vinal, 1986; Parul et al., 2014). A strong correlation between virulence of clinical isolates of $E$. coli and their expression on the CR agar medium was discovered by Berhkoff and Vinal (1986). So, the CR dye-binding could be applied as a phenotypic marker or virulence factor for pathogenic bacteria. A similar relationship between virulence and ability to bind to the CR dye was observed for other bacteria (Surgalla and Beesley, 1969; Payne and Finklestein, 1977; Prpic et al., 1983; Yoder, 1989). However, the exact mechanism of action of this test is unknown, but it is proposed that the presence of B-D-glycan in the bacterial cell wall may be involved (Vinal, 1988).

Table 1. Results of the bacteriological examination in head and body cellulitis lesions of broiler chickens in Egypt (March 2017 to March 2019)

\begin{tabular}{lccc}
\hline Site of lesion & $\begin{array}{c}\text { Number of } \\
\text { samples }\end{array}$ & $\begin{array}{c}\text { Number of samples positive } \\
\text { for bacterial culture (\%) }\end{array}$ & $\begin{array}{c}\text { Number of } \\
\text { bacterial isolates }\end{array}$ \\
\hline Head & 65 & $65(100 \%)$ & 129 \\
Thigh & 44 & $38(86.4 \%)$ & 41 \\
Abdomen & 71 & $67(94.4 \%)$ & 86 \\
Back & 27 & $25(92.6 \%)$ & 37 \\
Generalized & 83 & $76(91.6 \%)$ & 114 \\
Total Body & 225 & $206(91.5 \%)$ & 278 \\
Total & 290 & $271(93.4 \%)$ & 407 \\
\hline
\end{tabular}

Table 2. Bacterial species isolated from head and body cellulitis lesions of broiler chickens in Egypt (March 2017 to March 2019)

\begin{tabular}{|c|c|c|c|c|c|}
\hline \multirow{2}{*}{ Bacterial spp. } & \multicolumn{2}{|c|}{ Sample site } & \multirow{2}{*}{$\begin{array}{c}\text { Total } \\
\text { Number }(\%) \\
\end{array}$} & \multicolumn{2}{|c|}{ Congo red positive } \\
\hline & Head & Body & & Number & $\%$ \\
\hline E. coli & $\begin{array}{c}47 \\
(36.4 \%)\end{array}$ & $\begin{array}{c}137 \\
(49.3 \%)\end{array}$ & $\begin{array}{c}184 \\
(45.2 \%)\end{array}$ & 121 & $65.8 \%$ \\
\hline Staphylococcus & $\begin{array}{c}43 \\
(33.3 \%)\end{array}$ & $\begin{array}{c}92 \\
(33.1 \%)\end{array}$ & $\begin{array}{c}135 \\
(33.2 \%)\end{array}$ & 84 & $62.2 \%$ \\
\hline Clostridia & $\begin{array}{c}7 \\
(5.4 \%)\end{array}$ & $\begin{array}{c}15 \\
(5.4 \%)\end{array}$ & $\begin{array}{c}22 \\
(5.4 \%)\end{array}$ & 16 & $72.7 \%$ \\
\hline Aeromonas spp. & $\begin{array}{c}3 \\
(2.3 \%)\end{array}$ & $\begin{array}{c}2 \\
(0.7 \%)\end{array}$ & $\begin{array}{c}5 \\
(1.2 \%)\end{array}$ & 3 & $60 \%$ \\
\hline Enterobacter spp. & $\begin{array}{c}5 \\
(3.9 \%)\end{array}$ & $\begin{array}{c}8 \\
(2.9 \%)\end{array}$ & $\begin{array}{c}13 \\
(3.2 \%)\end{array}$ & 5 & $38.5 \%$ \\
\hline Proteus mirabilis & $\begin{array}{c}5 \\
(3.9 \%)\end{array}$ & $\begin{array}{c}13 \\
(4.7 \%)\end{array}$ & $\begin{array}{c}18 \\
(4.4 \%)\end{array}$ & 7 & $38.9 \%$ \\
\hline P. aeruginosa & $\begin{array}{c}6 \\
(4.6 \%)\end{array}$ & $\begin{array}{c}3 \\
(1.1 \%)\end{array}$ & $\begin{array}{c}9 \\
(2.2 \%)\end{array}$ & 9 & $100 \%$ \\
\hline Streptococcus spp. & $\begin{array}{c}13 \\
(10.1 \%)\end{array}$ & $\begin{array}{c}8 \\
(2.9 \%)\end{array}$ & $\begin{array}{c}21 \\
(5.1 \%)\end{array}$ & 7 & $33.3 \%$ \\
\hline Total & 129 & 278 & 407 & 252 & $61.9 \%$ \\
\hline
\end{tabular}




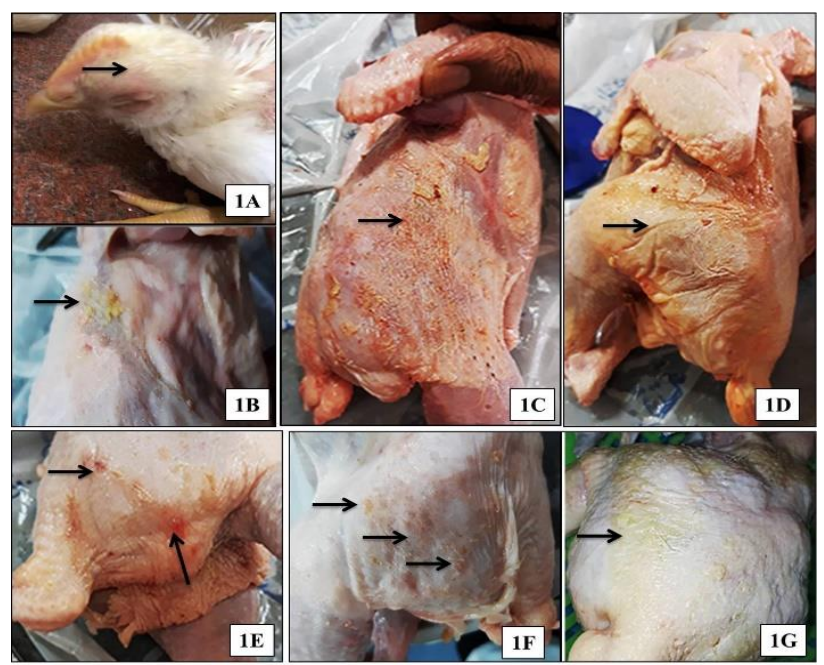

Figure 1. Macroscopic appearance of avian cellulitis at various body regions of broiler chickens. 1A: Swollen head (arrow); 1B: Irregular yellowish discoloration (arrow); 1C: Generalized brownish discoloration (arrow); 1D: Generalized dark yellow discoloration (arrow); 1E \& 1F: Localized circumscribed focal brownish discoloration (arrows), 1G: Focal asymmetrical yellow discoloration (arrow).

Table 3. Determination of serotypes of 184 E. coli isolates recovered from broiler chickens-affected cellulitis in Egypt (March 2017 to March 2019)

\begin{tabular}{lc}
\hline E. coli serotype (O group) & Number of isolates (\%) \\
\hline $\mathbf{O 1 1}$ & $7(3.8 \%)$ \\
$\mathbf{O 5 5}$ & $28(15.2 \%)$ \\
$\mathbf{O 7 8}$ & $35(19 \%)$ \\
$\mathbf{O 1 1 4}$ & $15(8.1 \%)$ \\
$\mathbf{O 1 2 5}$ & $9(4.9 \%)$ \\
$\mathbf{O 1 2 8}$ & $17(9.2 \%)$ \\
$\mathbf{0 1 4 6}$ & $13(7.1 \%)$ \\
$\mathbf{O 1 5 7}$ & $9(4.9 \%)$ \\
$\mathbf{O 1 5 8}$ & $33(17.9 \%)$ \\
un-typed & $18(9.8 \%)$ \\
\hline
\end{tabular}
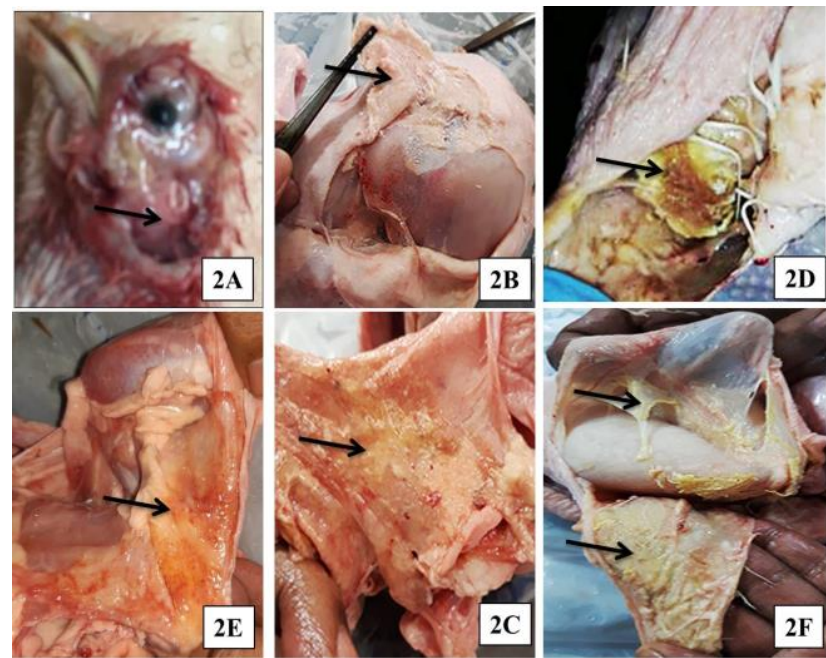

Figure 2. Macroscopic appearance of avian cellulitis after skinning in broiler chickens. 2A: Serosanguineous (arrow), 2B: Fibrinous subcutaneous exudate (arrow); 2E and 2C: Yellowish subcutaneous exudate (arrow); 2D: Greenish subcutaneous exudates (arrow); 2F: Suppurative subcutaneous exudate (arrow).

Table 4. The species of coagulase-positive and coagulasenegative staphylococci isolated from cellulitis lesions of broiler chickens in Egypt (March 2017 to March 2019)

\begin{tabular}{lcc}
\hline Staphylococci isolates & Staphylococcus spp. & $\begin{array}{c}\text { Number of } \\
\text { isolates (\%) }\end{array}$ \\
\hline Coagulase-positive & S. aureus & $43(55.1 \%)$ \\
Staphylococci isolates & S. intermedius & $15(19.2 \%)$ \\
(78 isolates) & S. hyicus & $9(11.5 \%)$ \\
& non-identified & $11(14.1 \%)$ \\
\hline & isolates & $8(14 \%)$ \\
Coagulase-negative & Sallinarum & $14(24.6 \%)$ \\
Staphylococci isolates & S. sciuri & $6(10.5 \%)$ \\
(57 isolates) & S. epidermidis & $7(12.3 \%)$ \\
& S. lentus & $11(19.3 \%)$ \\
& S. xylosus & $9(15.8 \%)$ \\
& S. haemolyticus & $2(3.5 \%)$ \\
\hline
\end{tabular}

In the present study, serotyping of isolated $E$. coli from cellulitis lesions revealed the presence of nine different $\mathrm{O}$ serotypes of E. coli, which O78 was the most predominant serotype (19\%) (Table 3). Similar results were obtained previously, where $E$. coli were isolated from cellulitis lesions and belonged to six O-groups, with $\mathrm{O} 78$ (52.2\%) being the most prevailing serotype (Derakhshanfar and Ghanbarpour, 2002). Noteworthy, O78 serotype of $E$. coli contains virulent strains related to severe infections in poultry. Also, serotype $\mathrm{O} 78$ has public health implications because it is considered one of the serotypes accompanied by enterotoxigenic E. coli strains that can infect humans directly by contact with infected birds (Messier et al., 1993).

The isolated staphylococci in this study were identified into three coagulase-positive staphylococci with $S$. aureus as the most prevalent strain $(55.1 \%)$, and seven coagulase-negative staphylococci in which S. sciuri was the most predominant strain $(24.6 \%$ ) (Table 4). Similarly, 17 Staphylococcus spp. (three coagulase-positive spp. and 14 coagulase-negative spp.) were isolated and identified from 100 cellulitis lesion samples in broiler chickens (Hilmy, 2002). Also, S. aureus was isolated from 12 out of 98 broiler carcasses with cellulitis (Derakhshanfar and Ghanbarpour, 2002).

Antibiotics are used in poultry farms as therapeutic agents and growth promoters, which have favorable and economic benefits for farmers. However, its excessive use is a big threat and results in emerging and dissemination of antibiotic-resistant strains of pathogenic and non-pathogenic organisms that could be transferred to humans via the food chain (Kariuki, et al., 1999; Apata, 2009; Suleiman et al., 2013). The rapid surge in the development and spread of antibiotic resistance is the main cause of concern (Aarestrup et al., 2008). Thus, testing isolated pathogens for antibiotic resistance has become a global interest in efficient preventive treatment and control measures. 
Table 5. The results of antibiotic susceptibility profile of bacterial pathogens isolated from cellulitis lesions of broiler chickens in Egypt (March 2017 to March 2019)

\begin{tabular}{|c|c|c|c|c|c|c|c|c|c|c|c|}
\hline \multirow{2}{*}{ Bacterial species } & \multirow{2}{*}{ State } & \multicolumn{10}{|c|}{ Antimicrobial agents } \\
\hline & & $\mathrm{CN}$ & OX & ERI & C30 & T30 & DA & ENR & AMP & CTX & VA \\
\hline \multirow{3}{*}{$\begin{array}{l}\text { E. coli } \\
(\mathbf{n}=184)\end{array}$} & $\mathbf{S}$ & $43(23.4 \%)$ & $16(8.7 \%)$ & $58(31.5 \%)$ & $22(11.9 \%)$ & $15(8.2 \%)$ & $181(98.4 \%)$ & $12(6.5 \%)$ & $29(15.8 \%)$ & $69(37.5 \%)$ & $23(12.5 \%)$ \\
\hline & $\mathbf{R}$ & $86(46.7 \%)$ & $137(74.5 \%)$ & $79(42.9 \%)$ & $153(83.1 \%)$ & $164(89.1 \%)$ & $2(1.1 \%)$ & $171(92.9 \%)$ & $124(67.4 \%)$ & $98(53.3 \%)$ & $138(75 \%)$ \\
\hline & I & $55(29.9 \%)$ & $31(16.8 \%)$ & $47(25.5 \%)$ & $9(4.9 \%)$ & $5(2.7 \%)$ & $1(0.5 \%)$ & $1(0.5 \%)$ & $31(16.8 \%)$ & $17(9.2 \%)$ & $23(12.5 \%)$ \\
\hline \multirow{3}{*}{$\begin{array}{l}\text { Staphylococcus } \\
\text { spp. } \\
(\mathbf{n}=135)\end{array}$} & $\mathbf{S}$ & $132(97.8 \%)$ & $28(20.7 \%)$ & $89(65.9 \%)$ & $105(77.8 \%)$ & $78(57.8 \%)$ & $9(6.7 \%)$ & $38(28.1 \%)$ & $4(2.9 \%)$ & $97(71.8 \%)$ & $47(34.8 \%)$ \\
\hline & $\mathbf{R}$ & $3(2.2 \%)$ & $103(76.3 \%)$ & $42(31.1 \%)$ & $29(21.5 \%)$ & $55(40.7 \%)$ & $112(82.9 \%)$ & $88(65.2 \%)$ & $131(97.0 \%)$ & $21(15.5 \%)$ & $84(62.2 \%)$ \\
\hline & I & $0(0 \%)$ & $4(2.97 \%)$ & $4(2.97 \%)$ & $1(0.7 \%)$ & $2(1.5 \%)$ & $14(10.4 \%)$ & $9(6.7 \%)$ & $0(0 \%)$ & $17(12.59 \%)$ & $4(2.9 \%)$ \\
\hline \multirow{3}{*}{$\begin{array}{l}\text { Clostridia spp. } \\
(\mathbf{n = 2 2})\end{array}$} & $\mathbf{S}$ & $3(13.6 \%)$ & $14(63.6 \%)$ & $20(90.9 \%)$ & $22(100 \%)$ & $0(0 \%)$ & $4(18.2 \%)$ & $0(0 \%)$ & $16(72.7 \%)$ & $0(0 \%)$ & $3(13.6 \%)$ \\
\hline & $\mathbf{R}$ & $18(81.8 \%)$ & $1(4.5 \%)$ & $2(9.1 \%)$ & $0(0 \%)$ & $22(100 \%)$ & $14(63.6 \%)$ & $22(100 \%)$ & $4(18.2 \%)$ & $22(100 \%)$ & $18(81.8 \%)$ \\
\hline & I & $1(4.5 \%)$ & $7(31.8 \%)$ & $0(0 \%)$ & $0(0 \%)$ & $0(0 \%)$ & $4(18.2 \%)$ & $0(0 \%)$ & $2(9.1 \%)$ & $0(0 \%)$ & $1(4.5 \%)$ \\
\hline \multirow{3}{*}{$\begin{array}{l}\text { Aeromonas spp. } \\
(\mathbf{n}=5)\end{array}$} & $\mathbf{S}$ & $0(0 \%)$ & $2(40 \%)$ & $2(40 \%)$ & $5(100 \%)$ & $0(0 \%)$ & $1(20 \%)$ & $0(0 \%)$ & $4(80 \%)$ & $0(0 \%)$ & $1(20 \%)$ \\
\hline & $\mathbf{R}$ & $3(60 \%)$ & $0(0 \%)$ & $3(60 \%)$ & $0(0 \%)$ & $5(100 \%)$ & $3(60 \%)$ & $5(100 \%)$ & $1(20 \%)$ & $5(100 \%)$ & $4(80 \%)$ \\
\hline & $\mathbf{I}$ & $2(40 \%)$ & $3(60 \%)$ & $0(0 \%)$ & $0(0 \%)$ & $0(0 \%)$ & $1(20 \%)$ & $0(0 \%)$ & $0(0 \%)$ & $0(0 \%)$ & $0(0 \%)$ \\
\hline \multirow{3}{*}{$\begin{array}{l}\text { Enterobacter spp. } \\
(\mathrm{n}=13)\end{array}$} & $\mathbf{S}$ & $11(84.6 \%)$ & $2(15.4 \%)$ & $2(15.4 \%)$ & $0(0 \%)$ & $8(61.5 \%)$ & $1(7.7 \%)$ & $3(23.1 \%)$ & $1(7.7 \%)$ & $0(0 \%)$ & $2(15.4 \%)$ \\
\hline & $\mathbf{R}$ & $2(15.4 \%)$ & $10(76.9 \%)$ & $8(61.5 \%)$ & $13(100 \%)$ & $3(23.1 \%)$ & $12(92.3 \%)$ & $8(61.5 \%)$ & $10(76.9 \%)$ & $13(100 \%)$ & $11(84.6 \%)$ \\
\hline & I & $0(0 \%)$ & $1(7.7 \%)$ & $3(23.1 \%)$ & $0(0 \%)$ & $2(15.4 \%)$ & $0(0 \%)$ & $2(15.4 \%)$ & $2(15.4 \%)$ & $0(0 \%)$ & $0(0 \%)$ \\
\hline \multirow{3}{*}{$\begin{array}{l}\text { Proteus mirabilis } \\
(\mathbf{n = 1 8})\end{array}$} & $\mathbf{S}$ & $7(38.8 \%)$ & $2(11.1 \%)$ & $4(22.2 \%)$ & $14(77.7 \%)$ & $6(33.3 \%)$ & $8(44.4 \%)$ & $5(27.7 \%)$ & $10(55.5 \%)$ & $13(72.2 \%)$ & $16(88.9 \%)$ \\
\hline & $\mathbf{R}$ & $10(55.5 \%)$ & $14(77.7 \%)$ & $14(77.7 \%)$ & $2(11.1 \%)$ & $12(66.7 \%)$ & $9(50 \%)$ & $9(50 \%)$ & $5(27.7 \%)$ & $5(27.7 \%)$ & $2(11.1 \%)$ \\
\hline & I & $1(5.6 \%)$ & $2(11.1 \%)$ & $0(0 \%)$ & $2(11.1 \%)$ & $0(0 \%)$ & $1(5.6 \%)$ & $4(22.2 \%)$ & $3(16.6 \%)$ & $0(0 \%)$ & $0(0 \%)$ \\
\hline \multirow{3}{*}{$\begin{array}{l}\begin{array}{l}\text { P. aeruginosa } \\
(\mathbf{n}=9)\end{array} \\
\end{array}$} & $\mathbf{S}$ & $4(44.4 \%)$ & $2(22.2 \%)$ & $0(0 \%)$ & $1(11.1 \%)$ & $0(0 \%)$ & $2(22.2 \%)$ & $0(0 \%)$ & $2(22.2 \%)$ & $3(33.3 \%)$ & $2(22.2 \%)$ \\
\hline & $\mathbf{R}$ & $5(55.5 \%)$ & $7(77.7 \%)$ & $8(88.8 \%)$ & $8(88.8 \%)$ & $9(100 \%)$ & $7(77.7 \%)$ & $9(100 \%)$ & $7(77.7 \%)$ & $5(55.5 \%)$ & $7(77.7 \%)$ \\
\hline & $\mathbf{I}$ & $0(0 \%)$ & $0(0 \%)$ & $1(11.1 \%)$ & $0(0 \%)$ & $0(0 \%)$ & $0(0 \%)$ & $0(0 \%)$ & $0(0 \%)$ & $1(11.1 \%)$ & $0(0 \%)$ \\
\hline \multirow{3}{*}{$\begin{array}{l}\text { Streptococcus spp. } \\
(\mathbf{n}=\mathbf{2 1})\end{array}$} & $\mathbf{S}$ & $3(14.3 \%)$ & $2(9.5 \%)$ & $0(0 \%)$ & $7(33.3 \%)$ & $3(14.3 \%)$ & $18(85.7 \%)$ & $5(23.8 \%)$ & $7(33.3 \%)$ & $9(42.8 \%)$ & $6(28.6 \%)$ \\
\hline & $\mathbf{R}$ & $18(85.7 \%)$ & $16(76.2 \%)$ & $21(100 \%)$ & $13(61.9 \%)$ & $15(71.4 \%)$ & $2(9.5 \%)$ & $14(66.7 \%)$ & $10(47.6 \%)$ & $7(33.3 \%)$ & $13(61.9 \%)$ \\
\hline & I & $0(0 \%)$ & $3(14.3 \%)$ & $0(0 \%)$ & $1(4.7 \%)$ & $3(14.3 \%)$ & $1(4.7 \%)$ & $2(9.5 \%)$ & $4(19.0 \%)$ & $5(23.8 \%)$ & $2(9.5 \%)$ \\
\hline
\end{tabular}

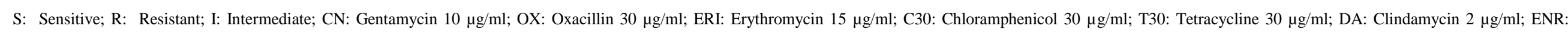
Enrofloxacin $5 \mu \mathrm{g} / \mathrm{ml}$; AMP: Ampicillin $10 \mu \mathrm{g} / \mathrm{ml}$; CTX: Cefatoxaime $30 \mu \mathrm{g} / \mathrm{ml}$; VA: Vancomycin $30 \mu \mathrm{g} / \mathrm{ml} \quad$ n: number 
Table 6. Antibiotic sensitivity patterns of bacterial spp. isolated from cellulitis lesions of broiler chickens in Egypt (March 2017 to March 2019)

\begin{tabular}{|c|c|c|c|c|c|c|c|c|c|c|c|}
\hline \multirow{2}{*}{$\begin{array}{l}\text { No. of antibiotics } \\
\text { to which the } \\
\text { organism is } \\
\text { resistant }\end{array}$} & \multirow{2}{*}{$\begin{array}{l}\text { Resistance } \\
\text { index }\end{array}$} & \multirow[b]{2}{*}{$\begin{array}{l}\text { Resistance } \\
\text { class }\end{array}$} & \multicolumn{9}{|c|}{ Distribution of bacterial spp. according to drug resistance index; $n(\%)$} \\
\hline & & & $\begin{array}{l}E . \text { coli } \\
\mathrm{n}=184\end{array}$ & $\begin{array}{c}\text { Staphylococcus } \\
\text { spp. } \\
\mathbf{n}=135\end{array}$ & $\begin{array}{c}\text { Clostridia } \\
\text { spp. } \\
\mathbf{n = 2 2}\end{array}$ & $\begin{array}{c}\text { Aeromonas } \\
\text { spp. } \\
\mathbf{n = 5}\end{array}$ & $\begin{array}{c}\text { Enterobacter } \\
\begin{array}{c}\text { spp. } \\
n=13\end{array}\end{array}$ & $\begin{array}{l}\text { Proteus } \\
\text { mirabilis } \\
\quad \mathrm{n}=18\end{array}$ & $\begin{array}{c}P \text {. aeruginosa } \\
\mathrm{n}=9\end{array}$ & $\begin{array}{c}\text { Streptococcus } \\
\text { spp. } \\
\mathbf{n = 2 1}\end{array}$ & $\begin{array}{c}\text { Total } \\
\text { isolates } \\
n=407\end{array}$ \\
\hline 0 & 0 & $\mathrm{~S}$ & $12(6.5 \%)$ & $4(2.9 \%)$ & $0(0 \%)$ & $0(0 \%)$ & $2(15.4 \%)$ & $0(0 \%)$ & $0(0 \%)$ & $0(0 \%)$ & $18(4.4 \%)$ \\
\hline $1-2$ & $0.1-0.2$ & NDR & $20(10.9 \%)$ & $\begin{array}{c}9 \\
(6.6 \%)\end{array}$ & $1(4.5 \%)$ & $0(0 \%)$ & $2(15.4 \%)$ & $2(11.1 \%)$ & $1(11.1 \%)$ & $3(14.2 \%)$ & $38(9.3 \%)$ \\
\hline $3-7$ & $0.3-0.7$ & MDR & $99(53.8 \%)$ & $82(60.7 \%)$ & $9(40.9 \%)$ & $3(60 \%)$ & $9(69.2 \%)$ & $10(55.5 \%)$ & $1(11.1 \%)$ & $16(76.1 \%)$ & $229(56.3 \%)$ \\
\hline $8-9$ & $0.8-0.9$ & XDR & $44(23.9 \%)$ & $27(20 \%)$ & $10(45.5 \%)$ & $2(40 \%)$ & $0(0 \%)$ & $5(27.8 \%)$ & $7(77.8 \%)$ & $2(9.5 \%)$ & $97(23.8 \%)$ \\
\hline 10 & 1 & PDR & $3(1.6 \%)$ & $3(2.2 \%)$ & $0(0 \%)$ & $0(0 \%)$ & $0(0 \%)$ & $0(0 \%)$ & $0(0 \%)$ & $0(0 \%)$ & $6(1.5 \%)$ \\
\hline
\end{tabular}

S: Sensitive; NDR: Narrow drug-resistant; MDR: Multidrug-resistant; XDR: Extensively drug-resistant; PDR: Pandrug-resistant 
The results of in vitro sensitivity testing of the isolated bacterial strains are presented in table 5. It was found that the antibiogram profile of $E$. coli isolates showed highest resistance rate to enrofloxacin (92.9\%), followed by tetracycline $(89.1 \%)$, chloramphenicol $(83.1 \%)$, vancomycin $(75 \%)$, oxacillin $(74.5 \%)$, ampicillin $(67.4 \%)$, cefotaxime (53.3\%), gentamycin (46.7\%) and erythromycin (42.9\%). High susceptibility and lowest resistance to clindamycin (98.4\%) were recorded. These findings are in line with a previous study that indicated the high prevalence of resistance in E. coli isolated from broiler chickens against 10 different antimicrobial agents including ampicillin (100\%), tetracycline $(93.1 \%)$, nalidixic acid $(84.5 \%)$, chloramphenicol $(84.5 \%)$, kanamycin $(69.0 \%)$, sulfamethoxazoletrimethoprim $(58.6 \%)$, cefotaxime $(58.6 \%)$, streptomycin $(50.0 \%)$, gentamicin $(48.3 \%)$, and ciprofloxacin $(41.4 \%)$ (Awad et al., 2016). In Egypt, another study reported that E. coli has variable sensitivity ranged from $14.3 \%$ to trimethoprim + sulfamethoxazole to $64.3 \%$ to clindamycin (Amer et al., 2017). Also, the resistance rate to oxacillin among the E. coli recovered from broilers ranged from 78.1\% to 100\% (Ahmed et al., 2013; Ibrahim et al., 2019).

The antibiogram profile of Staphylococcus spp. indicated high resistance rate to ampicillin (97.0\%) and clindamycin $(82.9 \%)$, followed by oxacillin $(76.3 \%)$, enrofloxacin $(65.2 \%)$, vancomycin $(62.2 \%)$ and tetracycline $(40.7 \%)$. On the other hand, the lower rates of resistance to erythromycin $(31.1 \%)$, chloramphenicol $(21.5 \%)$ and cefotaxime $(15.5 \%)$ were recorded. High susceptibility and lowest resistance to gentamycin were detected $(97.8 \%)$. Similarly, Bala et al. (2016) concluded that antibiotics such as oxytetracycline, oxacillin, and ampicillin have low activity against the tested isolates of Staphylococcus spp. Similar patterns of antimicrobial susceptibility have been previously reported (Pesavento et al., 2007; Otalu et al., 2011; Leonard and Markey, 2008; Waters et al., 2011). These findings may be a result of the extensive usage of these antimicrobial agents in animal husbandry over time, which has contributed to the development of drug-resistant strains (Nemati et al., 2008). On the other hand, Suleiman et al. (2013) reported $100 \%$ susceptibility to gentamicin and $66.7 \%$ to Augmentin.

The antibiogram profile of Clostridia isolates showed $100 \%$ resistance to tetracycline, enrofloxacin, and cefotaxime, as well as a high resistance rate of $81.8 \%, 81.8 \%$ and $63.6 \%$ to gentamycin, vancomycin, and clindamycin, respectively. However, these isolates showed low rates of resistance and high susceptibility to oxacillin (4.5\%), erythromycin $(9.1 \%)$, ampicillin $(18.2 \%)$ and complete sensitivity to chloramphenicol.

In Egypt, Osman and Elhariri (2013) recorded that clostridial isolates obtained from broiler flocks exhibited resistance toward gentamicin, streptomycin, oxolinic acid, lincomycin, erythromycin, and spiramycin. The prevalence of resistance to other antibiotics was also high, as doxycycline (98\%), trimethoprim-sulfamethoxazole (98\%), colistin (94\%), pefloxacin (94\%), neomycin (93\%), enrofloxacin (82\%), flumequine (78\%), oxytetracycline (71\%), norfloxacin (67\%), tylosin-fosfomycin (52\%), ciprofloxacin (58\%), spectinomycin (50\%), chloramphenicol (46\%), and rifampicin (34\%). The aforementioned study recommended drugs such as amoxicillin, ampicillin, cephradine, fosfomycin, and florfenicol for $C$. perfringens infection treatment in Egypt. Another study conducted in Korea recorded that $C$. perfringens isolated from chickens were susceptible to ampicillin, amoxicillin/clavulanic acid, cephalothin, cefepime, chloramphenicol, cefoxitin, ceftiofur, florfenicol, and penicillin but resistant to gentamycin, neomycin, streptomycin, apramycin and colistin (Park et al., 2015). This trend of resistance was similar to that detected in Taiwan by Fan et al. (2016) who found that most $C$. perfringens isolates from broiler chickens showed resistance against erythromycin, lincomycin, and chlortetracycline but susceptibility to amoxicillin, bacitracin, and enrofloxacin.

The result from the antibiogram profile of Aeromonas spp. revealed complete resistance to tetracycline, enrofloxacin, and cefotaxime, followed by vancomycin (80\%), gentamycin (60\%), erythromycin (60\%) and clindamycin $(60 \%)$. while low rates of resistance to ampicillin (20\%) and complete sensitivity to chloramphenicol were detected. These results were in accordance with that obtained by Ghenghesh et al. (2013) who reported Aeromonas isolated from chicken carcasses were susceptible to ciprofloxacin, ceftriaxone, and gentamicin. The isolates showed a significantly higher resistance rate to tetracycline. On the contrary, some studies reported complete resistance of Aeromonas to ampicillin and other penicillins (Ghenghesh et al., 2001; Hammad et al., 2018).

Antibiogram profile of Enterobacter spp. showed complete resistance to both chloramphenicol and cefotaxime, followed by clindamycin $(92.3 \%)$, vancomycin $(84.6 \%)$, oxacillin $(76.9 \%)$ and ampicillin $(76.9 \%)$, erythromycin (61.5\%), enrofloxacin (61.5\%), tetracycline $(23.1 \%)$ and gentamycin (15.4\%). Previous studies reported that Enterobacter spp. isolated from chickens were resistant to multiple antibiotics including ampicillin, cefotaxime, and gentamicin (Dennison and Morris, 2002; Kilonzo-Nthenge et al., 2008).

Antibiogram profile of Proteus mirabilis demonstrated $77.7 \%$ resistance to both oxacillin and erythromycin, followed by tetracycline $(66.7 \%)$, gentamycin $(55.5 \%)$, clindamycin $(50 \%)$, enrofloxacin (50\%), ampicillin $(27.7 \%)$, cefotaxime (27.7\%), chloramphenicol (11.1\%) and vancomycin (11.1\%). In a previous study by Nemati, (2013), Proteus isolates recovered from poultry were found to be highly resistant to nalidixic acid (93\%), doxycycline (91\%) and oxytetracycline (89\%). Moreover, low resistance to norfloxacin (24\%), ampicillin (22\%), ceftriaxone (22.4\%) and amikacin (24\%) and high susceptibility to gentamycin were reported (Nemati, 2013). In Bangladesh, a similar trend of antibiotic resistance was noticed in 36 Proteus isolates from chicken and 95\% of the isolates showed resistance against 
tetracycline, $89 \%$ against nalidixic acid and $20 \%$ were resistant against ciprofloxacin. Totally, $84 \%$ of the isolates exhibited MDR (Nahar et al., 2014).

The antibiogram profile of $P$. aeruginosa showed complete resistance to tetracycline and enrofloxacin, followed by erythromycin and chloramphenicol with the same resistance rate of $88.8 \%$. In addition, a resistance rate of $77.7 \%$ to oxacillin, clindamycin, ampicillin, and vancomycin was found. Also, gentamycin and cefotaxime had the same resistance rate of 55.5\%. In Pakistan, Sharma et al. (2017) investigated that P. aeruginosa isolates from chicken exhibited $100 \%$ resistance toward ceftriaxone, meropenem, ciprofloxacin, erythromycin, and colistin, while $60 \%$ sensitivity was noticed to ampicillin-sulbactam, ceftazidime, cefoperazone, and rifampicin. Isolates exhibited variable multidrug resistance patterns to other antibiotics. Another study carried out in Nigeria demonstrated that the P. aeruginosa isolates showed high resistance to $\beta$-lactams, tetracycline, tobramycin, nitrofurantoin, and sulfamethoxazole-trimethoprim, while ofloxacin, imipenem, and ertapenem appeared highly effective against the bacterial pathogens (Aniokette et al., 2016).

The antibiogram profile of Streptococcus spp. revealed complete resistance to erythromycin, followed by gentamycin $(85.7 \%)$, oxacillin $(76.2 \%)$, tetracycline $(71.4 \%)$, chloramphenicol (61.9\%), vancomycin (61.9\%), enrofloxacin (66.7\%), ampicillin (47.6\%), cefotaxime (33.3\%) and clindamycin (9.5\%). A study carried out in Japan reported that most of the examined Streptococcus isolates appeared susceptible to vancomycin, penicillin G and ampicillin, while some showed resistance to tetracycline, doxycycline, and lincomycin (Nomoto et al., 2013).

The misuse of antimicrobial at sub-therapeutic doses or unneeded doses contributes to the emergence of MDR bacteria (Yang et al., 2004). Concerning the result of MDR, it was demonstrated that only three bacterial spp., including Staphylococcus spp. (2.9\%), E. coli (6.5\%) and Enterobacter spp. (15.4\%), were sensitive to all tested antibiotics. All tested bacteria spp. had narrow drug-resistant isolates ranged from $4.5 \%$ to $15.4 \%$. E. coli (1.6\%) and Staphylococcus spp. (2.2\%) had isolates related to the PDR group. The prevalence of MDR, XDR, and PDR isolates was 56.3\%, 23.8\%, and $1.5 \%$, respectively (Table 6). Among the total of 407 bacterial isolates, 332 isolates (81.6\%) were found to be MDR with MDR Index $\geq 0.3$. A previous study by Xia et al. (2011) reported that over $58 \%$ of E. coli isolates showed resistance to four or more antimicrobial agents. The growing incidence of MDR is a public health issue due to the danger of entering the human food chain (Angulo et al., 2005).

In Egypt, 42\%-83.3\% of examined E. coli isolates were MDR to 5-10 out of 12 tested antibiotics (Amer et al., 2018). Another study reported a high prevalence of MDR as all examined E. coli isolates showed resistance to at least five anti-microbial agents (Ibrahim et al., 2019). The study of resistance patterns of the $C$. perfringens isolates indicated that all examined isolates exhibited resistance to 8-11 types of antibiotics and all were MDR (Osman and Elhariri, 2013). In Poland, over half of 302 Staphylococcus strains isolated from poultry were resistant to five of the used antibiotics, with the highest percentage recorded for enrofloxacin (Marek et al., 2016).

\section{CONCLUSION}

In conclusion, avian cellulitis had economic damage due to the high rate of carcass condemnation at slaughterhouses. $E$. coli serotype $\mathrm{O} 78$ as a zoonotic pathogen was the most predominant pathogen involved in cellulitis. A high prevalence of MDR among bacterial isolates was found, particularly against commonly used antibiotics. Therefore, it is recommended that the use of antimicrobial agents should follow prudent guidelines to minimize the development and spread of resistant bacteria. Also, the utilization of some antibiotics such as tetracycline, oxytetracycline, and erythromycin in poultry farms should be revised. Moreover, susceptibility testing should be performed to assure drugs of choice.

\section{DECLARATIONS}

\section{Acknowledgments}

The authors thank the Department of Poultry Diseases, Faculty Veterinary Medicine, Cairo University, and Poultry Diseases Department, Veterinary Research Division, National Research Centre, Giza, Egypt, for facilities during this study. This work was self-funded by team members and all authors declared that they did not receive any specific fund for this study.

\section{Competing interests}

The authors declare that they have no competing interests.

\section{Authors' contributions}

Mohamed M. Amer and Hoda M. Mekky designed the study, drafted and revised the manuscript. Hanaa S. Fedawy, Kh. M. Elbayoumi and Dalia M. Sedeek shared in samples collection, performing the tests, manuscript writing and data analysis. All authors read and approved the final manuscript. 


\section{REFERENCES}

Aarestrup FM, Wegener HC and Collignon P (2008). Resistance in bacteria of the food chain: epidemiology and control strategies. Expert Review of Anti-Infective Therapy, 6: 733-750. DOI: https://doi.org/10.1586/14787210.6.5.733

Ahmed AM, Shimamoto T and Shimamoto T (2013). Molecular characterization of multidrug-resistant avian pathogenic Escherichia coli isolated from septicemic broilers. International Journal of Medical Microbiology, 303(8): 475-483. DOI: https://doi.org/10.1016/j.ijmm.2013.06.009

Alves FM, Pereira VL, Nascimento ER, Guimarães AM, Almeida DO and Tortelly R (2007). Cellulitis associated with lesions of bursa of Fabricius from broilers under sanitary inspection. Revista Brasileira de ciência Veterinaria, 14(1): 23-27. Available at: https://www.researchgate.net/publication/284407244_Celulite_associada_as lesoes na bolsa_de_Fabricio de frangos de corte_ao abate_sob inspecao_sanitaria

Amer MM, ELbayoumi KhM, Girh ZMSA, Mekky HM and Rabie NS (2017). Study on bacterial contamination od dead in shell chicken embryos and culled one day chicks. International Journal of Pharmaceutical and Psychopharmacological Research, 7(2): 5-11. Available at: https://www.eijppr.com/en/article/a-study-on-bacterial-contamination-od-dead-in-shell-chicken-embryos-and-culled-one-day-chicks

Amer MM, Mekky HM, Amer AM and Fedawy HS (2018). Antimicrobial resistance genes in pathogenic Escherichia coli isolated from diseased broiler chickens in Egypt and their relationship with the phenotypic resistance characteristics, Veterinary World, 11(8): 1082-1088. DOI: https://dx.doi.org/10.14202\%2Fvetworld.2018.1082-1088

Amini K, Zachar T, Popowich S, Knezacek T, Goodhope B, Willson P and Gomis S (2015). Association of increased rate of condemnation of broiler carcasses due to hepatic abnormalities with immunosuppressive diseases in the broiler chicken industry in Saskatchewan. The Canadian Journal of Veterinary Research, 79: 261-267. Available at: https://www.ncbi.nlm.nih.gov/pmc/articles/PMC4581669/

Andrade LC (2005). Histopathology and identification of Escherichia coli as a causative agent of avian cellulitis in broiler chickens. Niteroi: Fluminense Federal University; 2005.

Angulo FJ, Collignon P, Wegener HC, Braam P and Butler CD (2005). The routine use of antibiotics to promote animal growth does little to benefit protein undernutrition in the developing world. Clinical Infectious Diseases, 41: 1007-1013. Available at: https://academic.oup.com/cid/article/41/7/1007/306577

Aniokette U, Iroha CS, Ajah MI and Nwakaeze AE (2016). Occurrence of multi-drug resistant Gram-negative bacteria from poultry and poultry products sold in Abakaliki. Journal of Agricultural Science and Food Technology, 2:119-124. Available at: https://pdfs.semanticscholar.org/c368/6280031c3aa1cdbc8ba95ca515015b98c710.pdf? ga=2.60601066.657548916.1573546863$\underline{1323428559.1573546863}$

Apata DF (2009). Antibiotic resistance in poultry. International Journal of Poultry Science, 8: 404-408. DOI: http://dx.doi.org/10.3923/ijps.2009.404.408

Awad A, Arafat N and Elhadidy M (2016). Genetic elements associated with antimicrobial resistance among avian pathogenic Escherichia coli. Annals of Clinical Microbiology and Antimicrobials, 15: 59. DOI: https://doi.org/10.1186/s12941-016-0174-9

Bala HK, Igwe JC, Olayinka BO, Olonitola OS and Onaolapo JA (2016). Molecular characterization of methicillin resistant S. aureus from poultry farms in Kano State, Nigeria. Journal of Bioscience and Biotechnology Discovery, 1(4): 74-82. DOI: https://doi.org/10.31248/JBBD2017.053

Barbieri NL, de Oliveira AL, Tejkowski TM, Pavanelo DB, Rocha DA, Matter LB, Callegari-Jacques SM, de Brito BG and Horn F (2013). Genotypes and pathogenicity of cellulitis isolates reveal traits that modulate APEC virulence. Plos One, 8(8):e72322. DOI: https://doi.org/10.1371/journal.pone.0072322

Barros LSS, Silva RM, Silva IM, Baliza MD and Virgílio FF (2013). Escherichia coli from cellulitis lesions in broilers. Journal of Food Measurement and Characterization, 7(1): 40-45. DOI: https://doi.org/10.1007/s11694-013-9138-3

Berkhoff HA and Vinal AC (1986). Congo red medium to distinguish between invasive and non- invasive Escherichia coli pathogenic for poultry. Avian Diseases, 30: 117-121. DOI: https://doi.org/10.2307/1590621

Bianco C, Balanescu B, Cieslicka U, Balanescu P, Stefanov K, Lopez P and Hristova N (2016). The Shades of avian cellulitis in meat-type chicken Journal of Veterinary Sciences, 2(2): 49-52. Available at: http://www.rroij.com/open-access/the-shades-of-avian-cellulitis-in-meattype-chicken.pdf

Brito KCT, Jaenisch and BG Brito (2011). Etiology of chicken cellulitis. XXII Latin American Poultry Congress 2011. Available at: https://en.engormix.com/poultry-industry/articles/cellulitis-in-poultry-t35092.htm

Christopher AF, Hora S and Ali Z (2013). Investigation of plasmid profile, antibiotic susceptibility pattern multiple antibiotic resistance index calculation of Escherichia coli isolates obtained from different human clinical specimens at tertiary care hospital in Bareilly-India. Annals of Tropical Medicine of Public Health, 6: 285-289. Available at: http://www.atmph.org/article.asp?issn=17556783; year $=2013$; volume $=6$; issue $=3$; spage $=285$; epage $=289$; aulast $=$ Christopher

CLSI (2016). Performance Standards for Antimicrobial Susceptibility Testing. 26th edition. CLSI supplement M100S. Wayne, PA: Clinical and Laboratory Standards Institute. Available at: http://ljzx.cqrmhospital.com/upfiles/201601/20160112155335884.pdf

Collee JG, Fraser AG, Marmion BP and Simmons A (1996). Practical Medical Microbiology.14 ${ }^{\text {th }}$ Edition. Chuechill, Livingstone.

de Brito BG, LC Gaziri and MC Vidotto (2003). Virulence factors and clonal relationships among Escherichia coli isolates isolated from broiler chickens with cellulitis. Infection and Immunity, 71(7): 4175-4177. DOI: https://dx.doi.org/10.1128\%2FIAI.71.7.4175-4177.2003

Dennison SK and Morris J (2002). Multiresistant Enterobacter sakazakii wound infection in an adult. Infections in Medicine, 19: 533-535. Available at: https://www.medscape.com/viewarticle/444864

Derakhshanfar A and Ghanbarpour R (2002). A study on avian cellulitis in broiler chickens. Veterinarski arhiv, 72(5): 277-284. Available at https://www.researchgate.net/publication/242523334_A study on avian_cellulitis in broiler_chickens

Edward PR and Ewing WH (1972). Identification of Enterobacteriaceae. $3^{\text {rd }}$ Edition. Burgess Publishing Co., Minneapolis. pp. 709.

Fallavena LCB, Moraes HLS, Salle CTP, Silva AB, Vargas RS, Nascimento VP and Canal CW (2000). Diagnosis of skin lesions in condemned or downgraded broiler carcasses - a microscopic and macroscopic study. Avian Pathology, 29(6):557-562. DOI: https://doi.org/10.1080/03079450020016797

Fan YC, Wang CL, Wang C, Chen TC, Chou CH and Tsai HJ (2016). Incidence and antimicrobial susceptibility to Clostridium perfringens in premarket broilers in Taiwan. Avian Diseases, 60(2): 444-449. DOI: https://doi.org/10.1637/11315-110915-Reg

Forbes BA, Sahm DF and Weissfeld AS (2002). Diagnostic microbiology. $11^{\text {th }}$ Edition. Mosby, Inc. USA.

Ghenghesh KS, El-Ghodban A, Dkakni R, Abeid S, Altomi A, Tarhuni A (2001). Prevalence, differentiation, haemolytic activity, and antibiotic susceptibility of aeromonads in untreated well water. Memórias do Instituto Oswaldo Cruz, 96: 169-173. DOI: http://dx.doi.org/10.1590/S0074$\underline{02762001000200006}$

To cite this paper: Amer MM, Mekky HM, Fedawy HS, Elbayoumi KhM and Sedeek DM (2019). Antibiotic Profile of Bacterial Species Isolated from Broiler Chicken 
Ghenghesh KS, El-Mohammady H, Levin SY and Zorgani A (2013). Antimicrobial resistance profile of Aeromonas species isolated from Libya. Libyan Journal of Medicine, 8: 21320-21321. DOI: https://dx.doi.org/10.3402\%2Fljm.v8i0.21320

Gomis S, Amoako AK, Ngeleka AM, Belanger L, Althouse B, Kumor L, Waters E, Stephens S, Riddell C, Porter A and Allan B (2002). Hislopathologic and bacteriologic evaluations of cellulitis detected in legs and caudal abdominal regions of turkeys. Avian Diseases, 46(1):192197. DOI: https://doi.org/10.1637/0005-2086\%282002\%29046\%5B0192\%3AHABEOC\%5D2.0.CO\%3B2

Gomis SM, Gomis AIU, Horadagoda NU, Wijewardene TG, Allan BJ and Potter AA (2000). Studies on cellulitis and other disease syndromes caused by Escherichia coli in broilers in Sri Lanka. Tropical Animal Health and Production, 32(6): 341-351. Available at: https://link.springer.com/content/pdf/10.1023/A:1005293400605.pdf

Greenwood D, Slack RC and Peutherer JF (2005). Medical microbiology. $16^{\text {th }}$ Edition.Churchill Livingstone China.

Hammad AM, Moustafa AH, Mansour MM, Fahmy BM, Hamada MG, Shimamoto T and Shimamoto T (2018). Molecular and phenotypic analysis of hemolytic Aeromonasas strains isolated from food in Egypt revealed clinically important multidrug resistance and virulence profiles. Journal of Food Protection, 81(6): 1015-1021. DOI: https://doi.org/10.4315/0362-028X.JFP-17-360

Hilmy JOI (2002). Bacteria associated with avian cellulitis in broiler chicken. [Master Thesis]. Faculty of Veterinary Medicine, Department of Microbiology, University of Khartoum. Available http://khartoumspace.uofk.edu/bitstream/handle/123456789/7938/Bacteria\%20Associated\%20With\%20Avian\%20Cellulitis\%20In\%20Broiler\% 20Chickens.pdf?sequence $=1 \&$ isAllowed=y

Ibrahim MS, Hussein AH, Eid AAM and Lebdah MA (2019). Molecular characterization of Escherichia coli strains causing respiratory signs in broiler chickens in Egypt. Zagazig Veterinary Journal, 47(2): 168-182. DOI: https://dx.doi.org/10.21608/zvjz.2019.10214.1026

Jorgensen JH and Ferraro MJ (2009). Antimicrobial susceptibility testing: a review of general principles and contemporary practices. Clinical Infectious Diseases, 49(11): 1749-1755. DOI: https://doi.org/10.1086/647952

Kariuki S, Gilks C, Kimari J, Obanda A, Muyodi J, Waiyaki P and Hart C (1999). Genotype analysis of E. coli strains from children and chicken living in close contact. Applied and Environmental Microbiology, 65(2): 472-476. Available at: https://www.ncbi.nlm.nih.gov/pmc/articles/PMC91049/

Kilonzo-Nthenge, Nahashon SN, Chen F and Adefope N (2008). Prevalence and antimicrobial resistance of pathogenic bacteria in chicken and guinea fowl. Poultry Science, 87: 1841-1848. DOI: https://doi.org/10.3382/ps.2007-00156

Konemann, EW, Allen SD, Schrechen WM, Berrjer PC and Winn WJP (1992). Colour Atlas and Textbook of Diagnostic Microbiology.4 ${ }^{\text {th }}$ Edition. J.B. Lippincott Co., Philadelphia, USA.

Krumperman PH (1983). Multiple antibiotic resistance indexing of Escherichia coli to identify high-risk sources of fecal contamination of foods. Applied Environmental Microbiology, 46: 165-170. Available at: https://www.ncbi.nlm.nih.gov/pmc/articles/PMC239283/

Leonard FC and Markey BK (2008). Meticillin-resistant Staphylococcus aureus in animals: a review. The Veterinary Journal, 175: 27-36. DOI: https://doi.org/10.1016/j.tvj1.2006.11.008

Magiorakos AP, Srinivasan A, Carey RB, Carmeli Y, Falagas ME, Giske CG, Harbarth S, Hindler JF, Kahlmeter G, Olsson-Liljequist B, Paterson DL, Rice LB, Stelling J, Struelens MJ, Vatopoulos A, Weber JT and Monnet DL. (2012). Multidrug-resistant, extensively drug-resistant and pandrug-resistant bacteria: an international expert proposal for interim standard definitions for acquired resistance. Clinical Microbiology and Infection, 18(3): 268- 281. DOI: https://doi.org/10.1111/j.1469-0691.2011.03570.x

Marek A, Stepień-Pyśniak D, Pyzik E, Adaszek Ł, Wilczyński J and Winiarczyk S (2016). Occurrence and characterization of Staphylococcus bacteria isolated from poultry in Western Poland. Berliner und Münchener tierärztliche Wochenschrift, 129(3-4):147-152. Available at: https://www.researchgate.net/publication/298158341_Occurrence_and_characterization_of_Staphylococcus_bacteria_isolated_from_poultry_in Western Poland

Messier S, Quessy S, Robinson Y, Devriese LA, Hommez J and Fairbrother JM (1993). Focal dermatitis and cellulitis in broiler chickens: bacteriological and pathological findings. Avian Diseases, 37: 839-844. Available at: https://www.jstor.org/stable/1592039?seq=1\#metadata_info tab contents

Morley AJ and Thomson DK (1984). Swollen-head syndrome in broiler chickens. Avian Diseases, 28(1): 238-243. Available at: https://www.jstor.org/stable/1590147

Nahar A, Siddiquee M, Nahar S, Anwar KS, Ali SI and Islam S (2014). Multidrug resistant Proteus mirabilis isolated from chicken droppings in commercial poultry farms: Bio-security concern and emerging public health threat in Bangladesh. Biosafety and Health Education, 2(2): 120125. DOI: https://doi.org/10.4172/2332-0893.1000120

Nemati M (2013). Antimicrobial resistance of Proteus isolates from poultry. European Journal of Experimental Biology, 3(6): 499-500. Available at: https://pdfs.semanticscholar.org/8199/8287be8e187c2dd8c91ddeeea6aa5a3664bf.pdf

Nemati M, Hermans K, Lipinska U, Denis O, Deplano A, Struelens M, Devriese LA, Pasmans F and Haesebrouck F (2008). Antimicrobial resistance of old and recent Staphylococcus aureus isolates from poultry: first detection of livestock associated methicillin-resistant strain ST398. Antimicrobial Agents and Chemotherapy, 52(10): 3817-3819. DOI: https://dx.doi.org/10.1128\%2FAAC.00613-08

Nomoto R, Tien LHT, Sekizaki T and Osawa R (2013). Antimicrobial susceptibility of Streptococcus gallolyticus isolated from humans and animals. Japanese Journal of Infectious Diseases, 66: 334-336. DOI: https://doi.org/10.7883/yoken.66.334

Norton RA. 1997. Avian cellulitis. World's Poultry Science Journal, 53: 337-349. DOI: https://doi.org/10.1079/WPS19970027

Osman, KM and El Hariri M (2013). Antibiotic resistance of Clostridium perfringens isolates from broiler chickens in Egypt. Revue Scientifique et Technique, 32(3): 841-850. DOI: http://dx.doi.org/10.20506/rst.32.2.2212

Otalu OJ, Kabir J, Okolocha EC and Umoh VJ (2011). Multi-drug resistant coagulase positive Staphylococcus aureus from live and slaughtered chickens in Zaria, Nigeria. International Journal of Poultry Science, 10(11): 871-875. DOI: https://doi.org/10.3923/ijps.2011.871.875

Paniago M (2009). Reduction of condemnations at processing plant through vaccination in the hatchery. CEVA Animal Health Asia Pacific, 27. Available at: https://pdfs.semanticscholar.org/eec3/644ac2c602f83ff2bed5d83afa0e5f2f7046.pdf

Park JY, Kim S, Oh JY, Kim HR, Jang I, Lee HS and Kwon YK (2015). Characterization of Clostridium perfringens isolates obtained from 2010 to 2012 from chickens with necrotic enteritis in Korea. Poultry Science, 94(6):1158-1164. DOI: https://doi.org/10.3382/ps/pev037

Parul, Bist B, Sharma B and Jain U (2014) Virulence associated factors and antibiotic sensitivity pattern of Escherichia coli isolated from cattle and soil, Veterinary World, 7(5): 369-372. DOI: https://doi.org/10.14202/vetworld.2014.369-372

Paul S, Bezbaruah RL, Roy MK and Ghosh AC (1997) Multiple antibiotic resistance (MAR) index and its reversion in Pseudomonas aeruginosa. Letters in Applied Microbiology, 24: 169-171. DOI: https://doi.org/10.1046/j.1472-765X.1997.00364.X

Payne SM and Finielstien RA (1977). Detection and differentiation of iron-responsive avirulent mutants on Congo red agar. Infection and Immunity, 18: 94-98. Available at: https://www.ncbi.nlm.nih.gov/pmc/articles/PMC421198/ 
Peighambari SM, Vaillancourt JP, Wilson RA and Gyles CL (1995): Characteristics of Escherichia coli isolates from avian cellulitis. Avian Diseases, 39: 116-124. Available at: https://www.jstor.org/stable/1591990?seq=1\#metadata info tab contents

Pesavento G, Ducci B, Comodo N and Lo Nostro A (2007). Antimicrobial resistance profile of Staphylococcus aureus isolated from raw meat: a research for methicillin resistant Staphylococcus aureus (MRSA). Food Control, 18: 196-200. DOI: https://doi.org/10.1016/j.foodcont.2005.09.013

Poulsen LL, Bisgaard M, Jørgensen SL, Dideriksen T, Pedersen JR and Christensen H (2018). Characterization of Escherichia coli causing cellulitis in broilers. Veterinary Microbiology, 225:72-78. DOI: https://doi.org/10.1016/j.vetmic.2018.09.011

Prpic JK, Robins-Browne RM and Davey RB (1983): Differentiation between virulent and avirulent Yersinia enterocolitica isolates by using Congo red agar. Journal of Clinical Microbiology, 18: 486-490. Available at: https://www.ncbi.nlm.nih.gov/pmc/articles/PMC270839/

Quinn PJ, Carter ME, Markey BK and Carter GR (1994). Clinical Veterinary Microbiology. Welfe Publishing, Mosbay. Year Book Europe Limited.

Quinn PJ, Carter ME, Markey BK, Donnoly WJ and Leonard FC (2002). Veterinary Microbiology and Microbial Diseases 166-1117 Osney Mead. Oxford First Ltd, Registered in the United Kingdom.

Randall CJ, Meakins PA, Harris MP and Watt DJ (1984). A new skin disease in broilers? The Veterinary Record, 114: 246. DOI: https://doi.org/10.1136/vr.114.10.246

Rosenberger K, Klopp S, Eckroade RJ and Krauss WC (1975). The role of the infectious bursal agent and several avian adenoviruses in the hemorrhagic-aplastic-anemia syndrome and gangrenous dermatitis. Avian Diseases, 19: 717-729. Available at: https://www.jstor.org/stable/1589185?seq=1\#page scan tab contents

Santos MM, Alcântara ACM, Perecmanis S, Campos A and Santana AP (2014). Antimicrobial resistance of bacterial strains isolated from avian cellulitis. Brazilian Journal of Poultry Science, 16(1):13-18. DOI: http://dx.doi.org/10.1590/S1516-635X2014000100002

Sharma S, Galav V, Agrawal M, Faridi F and Kumar B (2017). Multi-drug resistance pattern of bacterial flora obtained from necropsy samples of poultry. Journal of Animal Health and Production, 5: 165-171. DOI: http://dx.doi.org/10.17582/journal.jahp/2017/5.4.165.171

Shawki MM, Lebdah MA, AM Shahin and SA Nassif (2017). Some studies on swollen head syndrome in broiler chickens in Egypt. Zagazig Veterinary Journal, 45(S1): 132-141. DOI: http://dx.doi.org/10.5281/zenodo.1218798

Sneath PHA, Mair NS, Sharpe ME and Holt JG (1986). Bergey's Manual of Systematic Bacteriol. Vol. 2.Williams and Wilkins Co. Baltimore.

Suleiman A, Zaria LT, Grema HA and Ahmadu P (2013). Antimicrobial resistant coagulase positive Staphylococcus aureus from chickens in Maiduguri, Nigeria. Sokoto Journal of Veterinary Sciences, 11(1): 51-55. DOI: http://dx.doi.org/10.4314/sokjvs.v11i1.8

Surgalla MJ and Beesley ED (1969): Congo red agar plating medium for detecting pigmentation in Pasteurella pestis. Applied Microbiology, 18: 834837. Available at: https://www.ncbi.nlm.nih.gov/pmc/articles/PMC378096/

Vieira TB, Franco RM, Magalhães H, Praxedes CS and Tortelly R (2006). Cellulitis in broilers slaughtered under sanitary inspection: gross and histopathological lesions associated with isolation of Escherichia coli. Revista Brasileira de Ciência Veterinária, 13(3): 174-177. DOI: http://dx.doi.org/10.4322/rbcv.2014.388

Vinal AC (1988). The Association of Congo red binding and virulence in Escherichia coli pathogenic for poultry. Dissertation Abstracts International, 49(6): 2059-2065.

Wang C, Macklin KS, Krehling JT and Norton RA (2005). Influence of infectious bursal disease and chicken anemia vaccines on the development of cellulitis and myositis lesions in cage-reared broilers. Journal of Applied Animal Research, 27: 65-69. DOI: https://doi.org/10.1080/09712119.2005.9706542

Waters AE, Contente-Cuomo T, Buchhagen J, Liu CM, Watson L, Pearce K, Foster JT, Bowers J, Driebe EM, Engelthaler DM, et al. (2011). Multidrug-resistant Staphylococcus aureus in US meat and poultry. Clinical Infectious Diseases, 52: 1227-1230. DOI: https://doi.org/10.1093/cid/cir181

Watts JL (2008). Clinical and laboratory standards institute performance standards for antimicrobial disk and dilution susceptibility tests for bacteria isolated from animals: Approved standard. National Committee for Clinical Laboratory Standards. Available at: https://www.dbt.univr.it/documenti/OccorrenzaIns/matdid/matdid485539.pdf

Xia X, Meng J, Zhao S, Bodeis-Jones S, Gaines SA, Ayers SL and Mcdermott PF (2011). Identification and antimicrobial resistance of extraintestinal pathogenic Escherichia coli from retail meats. Journal of Food Protection, 74: 38-44. DOI: https://doi.org/10.4315/0362-028X.JFP-10-251

Yang H, Chen S, White DG, Zhao S, McDermott P, Walker R and Meng J (2004). Characterization of multiple-antimicrobial-resistant Escherichia coli isolates from diseased chickens and swine in China. Journal of Clinical Microbiology, 42: 3483-3489. DOI: https://doi.org/10.1128/JCM.42.8.3483-3489.2004

Yoder WH Jr (1989). Congo red binding by Escherchia coli isolates from chickens. Avian Diseases, 33: 502-509. Available at: https://www.jstor.org/stable/1591112?seq=1\#page_scan_tab_contents 